\title{
Numerical Investigation of Finned-tube Heat Exchanger with Circular, Elliptical \& Rectangular Tubes
}

\author{
MD. HASIBUL HASAN ${ }^{1}$ \\ Department of Mechanical Engineering \\ Khulna University of Engineering \& Technology \\ Khulna, BANGLADESH \\ DIPAYAN MONDAL ${ }^{2}$ \\ Department of Mechanical Engineering \\ Khulna University of Engineering \& Technology \\ Khulna, BANGLADESH
}

\begin{abstract}
A three dimensional numerical study has been conducted on fi nned-tube heat exchanger with multiple rows of tubes using ANSYS (Fluent). The objective of this study is to numerically investigate finned tube heat exchanger with different ty pe of tubes such as circular, elliptical an d rectangular tubes. As circular tubes has much pressure drop so elli ptical and recta ngular tubes has been introduced $\mathrm{i} n$ order to re duce pressure drop. As well as heat transfer has also been examined. The finite volume bas ed CFD code ANS YS Fluent 16.2 is used to calculate the flow and temperature fields and by applying SIMPLEC algorithm. At low velocity of air and water, nothin $g$ significant occurred for the co mbination of tubes. At high velocit $y$ in maximum tube combination there was heat transfe $r$ (HT) enhance ment and pressure drop reduction when compared with circular tubes only in case of air. When the combinations of circular, elliptical and rectangular tubes has been compared with circular tube heat exchanger (CTHX) heat transfer reduces as well as pressure drop (PD) also reduces for air. In case of water vapor HT and PD behaves the same. When those combinations has been compared with elliptical tube HX, for air in some cases heat transfer remains same and on other case it increases. For pressure drop in case of air, in some cases it reduces and on other cases it reduces. For elliptical tube HX for the fluid water vapor HT and PD both remains same or reduces. This work has not been with conducted any numerical simulation on rectangular Heat exchanger reason behind i $t$ there isn't an $y$ existence of this kind of $h$ eat exchanger. However, it could be nu merically conducted to examine the results between those combination and rectangular heat exchanger.
\end{abstract}

Keywords: Conventional and m odified heat exchanger; Darc y friction factor; Fin and tube Heat exchan ger; Heat Transfer; Numerical simulation.

Received: October 11, 2019. Revised: March 9, 2020. Accepted: April 5, 2020. Published: April 30, 2020.

\section{NOMENCLATURE}

\begin{tabular}{|c|c|}
\hline B & $\begin{array}{l}\text { Baseline or Conventional heat } \\
\text { exchanger }\end{array}$ \\
\hline$C_{p}$ & Specific heat capacity in $\mathrm{J} / \mathrm{Kg} \mathrm{K}$ \\
\hline$D$ & Diameter of the circular tube in $\mathrm{m}$ \\
\hline$f$ & Darcy Friction factor \\
\hline$H$ & Fin Height in $\mathrm{m}$ \\
\hline$h$ & heat transfer co-efficient in $\mathrm{W} / \mathrm{m}^{2} \mathrm{k}$ \\
\hline$k$ & Thermal conductivity in $\mathrm{W} / \mathrm{mk}$ \\
\hline$L$ & Length of fin in $\mathrm{m}$ \\
\hline$m$ & Mass flow rate of air in $\mathrm{Kg} / \mathrm{s}$ \\
\hline M & Modified Heat Exchanger \\
\hline
\end{tabular}

\section{Introduction}

Plate fin heat exchangers consists of a number of corrugated metal plate $\mathrm{s}$ in $\mathrm{m}$ utual contact, each plate having four ap paratus serving a s inlet and outlet ports, and seal s designed to direct the fluids in alternate flow passage. The plates a re clamped

$\begin{array}{ll}N & \text { Number of tube rows } \\ \mathrm{Nu} & \text { Nusselt number } \\ \mathrm{Pr} & \text { Prandtl number } \\ \mathrm{Q} & \text { Heat transfer rate in } \mathrm{W} \\ \mathrm{Re} & \text { Reynolds number } \\ T & \text { Temperature in } \mathrm{K} \\ U & x \text {-component velocity at inlet in } \mathrm{m} / \mathrm{s} \\ \Delta p & \text { Pressure drop in } \mathrm{N} / \mathrm{m}^{2} \\ \Delta T l m & \text { Logarithmic mean temperature }\end{array}$

together in a frame that includes connections for the fluids. Since each plat e is generally provided peripheral gasket to provi de sealing arrangem ent [1]. The present analy sis is focused on plate fin and three different ty pe of tubes such as circular, elliptical \& rectangular tube. Air is passed in between plate fin and tubes for co mbination of 
tube. In this present analysis, fluid strea ms remain separate and the heat transfer $t$ akes place continuously through a dividing impervious wall. In cross flow exchanger, two fluid are norm al to each other and both fluids are unmixed. For unmixed-unmixed case, fluid tem perature variations are idealized as two-dimensional only for the inlet and outlet section [ 2]. Thermal effectiveness for the cro ss flow he at exchanger falls in between parallel flow and co unter flow arrangement. In many cases one gas is compressed to increase pressure so the density is high while the other at lower pressure and low density. Compared to liquid-liquid exchangers, the size of gas-gas exchanger is much larger, because the convective heat transfer co-efficient on the gas side is low compared to the liquid side [3]. Cir cular tube is used in conventional heat exchanger. But now a days, elliptical tube is introduced. Elliptical tube is used due to its good aerodynam ic shape. Elliptical tube has less cross sectional area than circular one. Influence of aerodynamic shape of an elliptical tube on the thermo-hydraulic performance is found quite significant by reducing the ellipticity ratio. Elliptical tubes also helps to reduce drag force [4]. Rectangular tube in heat exchanger is a new concept. This type of tube is used as a duct in air conditioning system. It is used to increase the heat transfer area which additionally enhance heat transfer. In this present analy sis, fluid strea ms remain separate and the heat transfer takes place continuously through a dividing impervious wall.

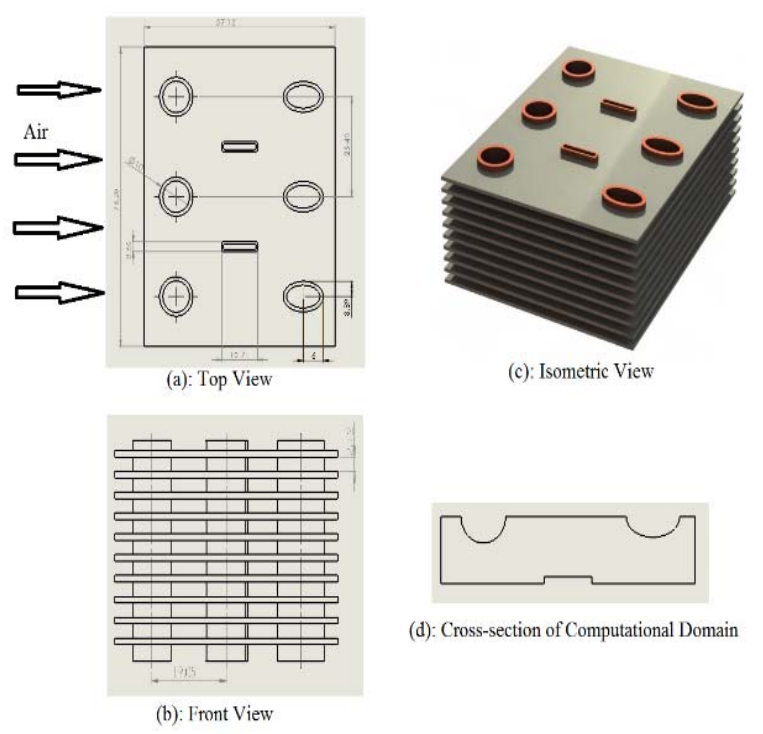

Fig 1: Schematic configuration of a heat exchanger for N3M1

\section{Literature Review}

Based on the intersecti on angle be tween the velocity and temperature gradient, Guo et al. [5 ] have proposed an approach to identif $y$ the region where the heat transfer should be enhanced in parabolic type flows. Later Based on field synergy principle $\mathrm{Li}$ et al. [6] have studied the fluid flow and heat transfer characteristics of strip fin w.r.t its position (upstream, downstream) and observed that strip fin in downstream arrangem ent performs better than o thers. A significant contribution on heat transfer enhancement and the stud y on flow characteristics of flow past various tube arrangements has been reported Jay avel and Tiwari et al. [7-9 ]. It is noticed that for the same heat transfer area using di fferent diameter of tube in the succes sive row y ields better performance over the sam e diameter tube. A m ultiple correlations of friction factor and Nusselt num ber for large tube diameter and large number of rows have been proposed by $\mathrm{Wu}$ and Tao at el[10], ], it is noticed that for the same heat transfer area using different diameter of tube in the succ essive row yields better performance over the same diameter tube. A multiple correlations of friction factor and Nusselt number for large tube diameter and large number of rows have been proposed by Xie at el[11]. The geometric effects on the pe rformance of plate finned-tube heat exchanger with different fin pitch, tube pitch, fin thickness, and tube diameter have been investigated by Lu et al [12 ]. From their work it is noticed that, with a fixed inlet frontal velocity there is enhancement in heat transfer by decrea sing tube diameter. The influence of tube shape on the therm o-hydraulic performance is found quit e significant fro $\mathrm{m}$ the work of Lotfi et al. [ 13], it is identifie d that the heat transfer performance is enhanced by reducing the tube ellipticity ratio.

\section{Theoretical Aspects}

\subsection{Flow across tube banks}

Because many heat-exchanger arr angements involve multiple rows of tubes, the heat transfer characteristics for tube banks are o $f$ important practical interest. The hea t-transfer characteristics of staggered and in-line tube banks were studied, and on the basis of a correlation of the results of various investigators. The original dat a were for gases with $\mathrm{Pr} \sim 0.7$. To extend the use to liquids, the present writer has modified the constants by the same $1.11 \mathrm{Pr}^{1 / 3}$ factor employed. The Reynolds number is based on the maximum velocity occurring in the tube bank; that is, the velocity 
through the mini mum-flow area. This area will depend on the geometric tube arrangement.

Heat transfer in tube banks can be computed from the general correlation [14]:

$$
N u_{D}=C_{1} C_{2} \operatorname{Re}^{n} \operatorname{Pr}^{m}\left(\frac{P r}{P r_{s}}\right)^{0.25}
$$

\subsection{Physical Model}

In this present sim ulation, a three dimensional numerical study of overall performance of the heat exchanger has been perfo rmed with co mbination of circular, elliptical and rectangular tube. Platefin with the staggered tube arrangement is taken to study with water-vapor and airside h eat transfer and pressure drop cha racteristics. The tube geometries considered are circular, elli ptical and combination of circular, elliptical and rectangular tube. Too closely represent the real time heat exchanger, multiple tube rows (number of tube rows, $\mathrm{N}=3,6$ ) are co nsidered. The effe ct of number of tu bes has al so been studied. Increas ed number of tu be leads to $d$ ecrease in he at transfer and when the nu mber exceeds 6, the corresponding increase in heat transfer i $\mathrm{s}$ negligible [4], therefore present work concerns up to six rows of tube.

The circular and elliptical tubes are designed in such a way that perimeter of the tubes are sa me, which allows ease of manufacturing and ensures the same heat transfer area for circular, elliptical and rectangular tube. Eccentricit y of the elliptical tube is taken as which is closer to Rocha at el [15]. The range of the inlet velocity is take $n$ between $0.5-2.5 \mathrm{~m} / \mathrm{s}$ with 0.5 intervals assu ming the flow to be laminar. As the region of interest of the present work is focused on to identify the tube geometric effect, the fin surface is assumed to be a constant wall temperature.

\subsection{Geo-metric Details}

Perimeter of all tube e.g . circular, elliptical and rectangular is taken as equal. For an ellipse having semi axes of lengths a and $b$, the perimeter $(P)$ is approximately [16].

$$
P=4 \times(a+b) \times\left(\frac{\pi}{4}\right)^{\left(\frac{4 a b}{(a+b)^{2}}\right)}
$$

Where, $a=6 \mathrm{~mm}$ and $b=3.89 \mathrm{~mm}$ by trial and error method taking eccentricity $e=b / a=0.6$ for rectangular tube perimeter is $=2 \times(a+b) a=$ $10.71 \mathrm{~mm}$ and $b=2.5 \mathrm{~mm}$

Perimeter of the circular tube $=2 \pi \mathrm{r}$; where radius of the circular tube $=5 \mathrm{~mm}$
Table 1: Geometrical data of the simulation.

\begin{tabular}{|l|c|}
\hline Transverse Pitch $\left(P_{t}\right)$ & $24.7 \mathrm{~mm}$ \\
\hline Longitudinal Pitch $\left(P_{l}\right)$ & $19.05 \mathrm{~mm}$ \\
\hline Circular Tube Diameter $(D)$ & $10 \mathrm{~mm}$ \\
\hline $\begin{array}{l}\text { Perimeter of circular, } \\
\text { elliptical \& rectangular tube }\end{array}$ & $31.42 \mathrm{~mm}$ \\
\hline Fin Height $(H)$ & $2.115 \mathrm{~mm}$ \\
\hline Number of Rows & 3,6 \\
\hline
\end{tabular}

Table 2: Combination of tube in various arrangement.

\begin{tabular}{|c|c|c|}
\hline $\begin{array}{c}\text { Designati } \\
\text { on }\end{array}$ & Schematic Representation & Category \\
\hline N3B1 & & $\begin{array}{c}\text { Baseline- } \\
1\end{array}$ \\
\hline N3B2 & & $\begin{array}{c}\text { Baseline- } \\
2\end{array}$ \\
\hline N3M1 & & $\begin{array}{c}\text { Modified- } \\
1\end{array}$ \\
\hline N3M2 & & $\begin{array}{c}\text { Modified- } \\
2\end{array}$ \\
\hline N3M3 & & $\begin{array}{c}\text { Modified- } \\
3\end{array}$ \\
\hline N3M4 & & $\begin{array}{c}\text { Modified- } \\
4\end{array}$ \\
\hline N3M5 & & $\begin{array}{c}\text { Modified- } \\
5\end{array}$ \\
\hline N3M6 & & $\begin{array}{c}\text { Modified- } \\
6\end{array}$ \\
\hline N6B1 & & $\begin{array}{c}\text { Baseline- } \\
1\end{array}$ \\
\hline N6B2 & & $\begin{array}{c}\text { Baseline- } \\
2\end{array}$ \\
\hline N6M1 & & $\begin{array}{c}\text { Modified- } \\
1\end{array}$ \\
\hline N6M2 & & $\begin{array}{c}\text { Modified- } \\
2\end{array}$ \\
\hline N6M3 & & $\begin{array}{c}\text { Modified- } \\
3\end{array}$ \\
\hline N6M4 & & $\begin{array}{c}\text { Modified- } \\
4\end{array}$ \\
\hline N6M5 & & \\
\hline N6M6 & & \\
\hline
\end{tabular}

\subsection{Mesh Generation and Solution Methodology}

The computational domain is discreti zed into a finite number of control volum e. In fin region, inflation mesh control is done with four edges of circular and elliptical in both sides. First lay er thickness inflation option is sele cted. After that body sizing is done in fi $\mathrm{n}$ region. In upstream and 
downstream region. Several edge sizing is done in various direction.

The finite volume based CFD code ANSYS Fluent 16.2 is used to calculate the flow and te mperature fields and by applying SIMPLEC algorithm. Under relaxation factor for pressure correction is taken as 1 for faster convergence [16]. To obtain improved accuracy of the solution, second order spatial discretization of the press ure is employed. As the grids are structured hexa hedral and a re aligned with flow direction, QUICK scheme is used for discretizing higher order convective terms in momentum equation. The residual is $10^{-6}$ for continuity and $\mathrm{m}$ omentum, whereas for energy equation, it is taken as $10^{-8}$.

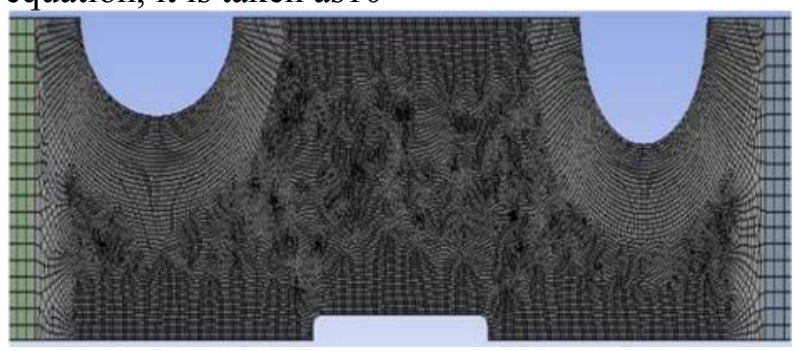

Fig 2: Schematic representation of grid.

\subsection{Parametric Definition}

The definitions of non- dimensional parameters such as Reynolds number $(R e)$, Nusselt num ber $(\mathrm{Nu})$ and Darcy friction factor $(f)$ are defined as follows [9],

$$
L M T D=\frac{\left(T_{w}-T_{\text {in }}\right)-\left(T_{w}-T_{\text {out }}\right)}{\ln \frac{\left(T_{w}-T_{\text {in }}\right)}{\left(T_{w}-T_{\text {out }}\right)}}
$$

Heat Transfer rate

$$
\begin{gathered}
Q=m C_{p}\left(T_{\text {out }}-T_{\text {in }}\right) \\
h_{m}=\frac{Q}{L M T D \times A_{s}} \\
R e=\frac{\rho U \text { inH }}{\mu} \\
N u=\frac{h H}{k} \\
f=\frac{\Delta P}{0.5 \rho{U_{\text {in }}}^{2}} \frac{H}{L}
\end{gathered}
$$

\section{Numerical Results and Discussion}

\subsection{Mesh Sensitivity}

For checking mesh dependency baseline N3B1 is taken for consideration. Grid $1=264935 \mathrm{n}$ odes, Grid $2=361911$ nodes, Grid $3=498982$ nodes. Mesh dependency is checked for heat tr ansfer coefficient at different inlet velocity. From Fig 4.1, it is observed that at grid 1 and grid 3 gives alm ost same heat transfer $\mathrm{c}$ o-efficient. For further calculation, grid 1 is taken as best option considering time for solution to converge.

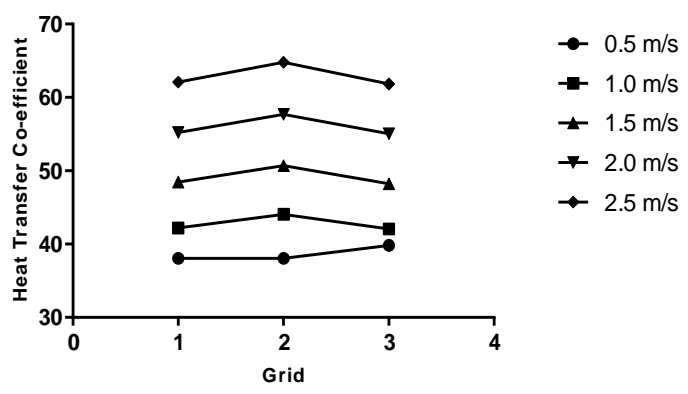

Fig 3: Grid independence results $(\mathrm{N}=3)$

\subsection{Validation of Numerical Results}

For the fin and tube heat exchangers with plain fin configuration, the air side performance characteristics have been exa mined experimentally for various sam ples (varying geometrical parameters) [17] The present results are validated with the experimental work of Wang at el. [17] and a close agreement has been observed as shown in Fig 4

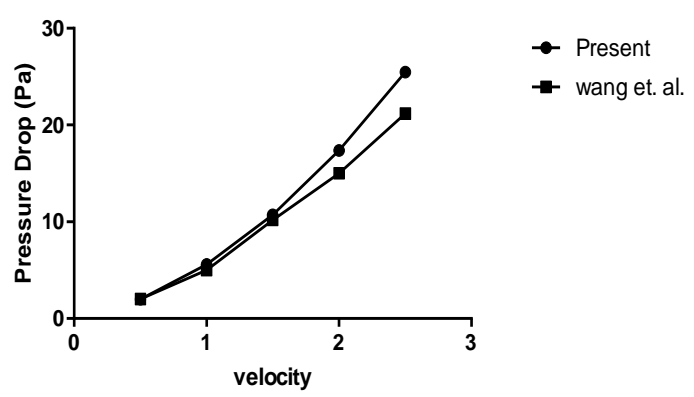

Fig 4: Validation results for $\mathrm{N}=\mathbf{2}$

\subsection{Heat Transfer of Air}

The connection of all tubes may pose manufacturing difficulty, but wit $\mathrm{h}$ advanced manufacturing technology, this connection is quite possible if all tubes are maintained a $t$ the sa me perimeter [16].

The result has been prese nted on Normalized $\mathrm{Nu}$ number and friction factor. The results are normalized $(\mathrm{Xi} / \mathrm{Xo})$, where i stands for modified cases and o stands for ba seline cases which are conventional method such as heat ex changer with circular and elliptical tube rows. The hy draulic diameter of circular and elliptical tubes are different due to differences in cross sect ion under constant perimeter, hence, Reynolds number based on fin height is used for representing the results.

From Fig 5, it shows that N3M1 perfo rms better than N3M2 when compared to N3B1. Though heat transfer gradually decreases, but at lower inlet velocities both perform better than convention grouped circular tubes Circular tube $h$ as a higher 
heat transfer rate than elliptical and rectangular tubes. Heat transfer for ell iptical tube almost half circular tubes. At lower inlet velocity heat transfer of $\mathrm{N} 3 \mathrm{M} 1$ is greater than $\mathrm{N} 3 \mathrm{~B} 1$ due to no circulation occurs at this velocity . Mostly heat transfer by diffusion in this low velocity. And in this case for ced convection to takes place. In contrast, at high Re num ber N3M4 performs quite better than N3M3. N3M5 and N3M6 acts al most in similar fashion when compared with N3B1.N3M5 and N3M6 also acts almost similar fashion when co mpared with N3B2. N3M6 is a better option than N3M5. From Fig 6, the curve is upward when compared with N6B1. But N6M1 is better than N6M2 at hi gh Re num ber. But for N6M2 almost all the tube gives higher circulation. For this reason high heat transfer for N6M1 than N6M2. In both cases, as velocity increases heat transfer increases, but heat transfer rate of N6B1 is much higher than N6M3. N6M4 also acts like N6M3 when co mpared with N6B1. N6M3 when compared with N6B2 it was seen the heat transfer for both of the cases are almost similar. N6M5 and N6M6 acts si milar when compared with N6B1. But at high inlet velocity, heat transfer for both cases increases.

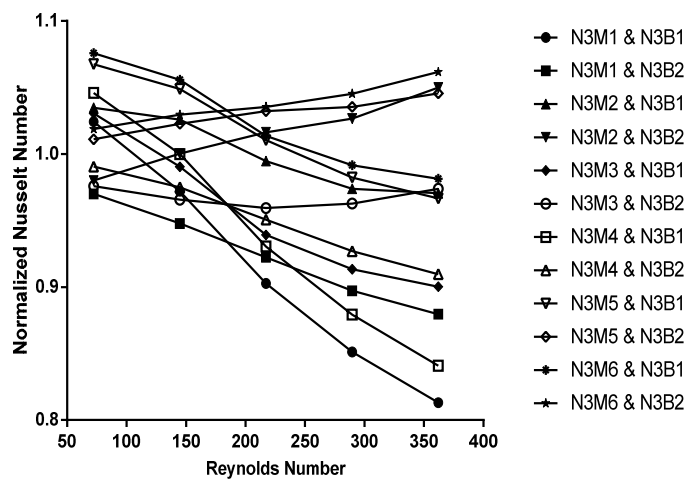

Fig 5: Nusselt Number of air of Modified Heat Exchanger (HX) with corresponding Reynolds Number of Conventional $\mathrm{HX}$ for $\mathrm{N}=\mathbf{3}$

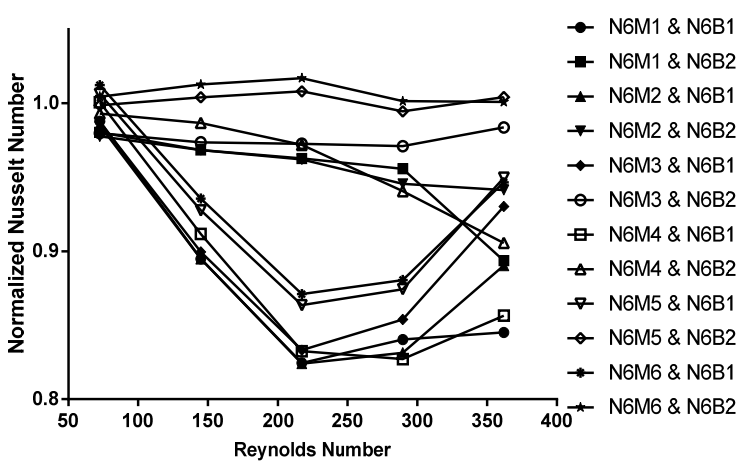

Fig 6: Nusselt Number of air of Modified Heat Exchanger (HX) with corresponding Reynolds Number of Conventional $\mathrm{HX}$ for $\mathrm{N}=6$

\subsection{Heat Transfer rate for water-vapor}

Water vapor is also taken in steads of air with all those combinations. Due to having different properties of water vapor there is dif ference in heat transfer and Nusselt number.

From Fig 7, N3M2 is a b etter option than N3M2 for water-vapor. But in reverse action is shown for N3M2 when com pared with N3B2. For watervapor N3M3 and N3M4 both have a low heat transfer rate from low inlet velocity to the high inlet velocity when co mpared with N3B1. Similar things happen for N3M3 and N3M4 when compared with N3B2. N3M5 \& N3M6 combinations are bett er than the previous two named as N3M3 and N3M4. For water-vapor N3M5 and N3M6 both have a low heat transfer rate from low inlet velo city to the high inle $t$ velocity when com pared with N3B1. Sim ilar things happen for N3M5 and N3M6 when compared with N3B2.

From Fig 8, N6M1 and N6M2 are co mpared with N6B1, at that time heat tr ansfer is so much lower in high inlet velocity. But N6M1 and N6M2 are compared with N6B2 which is b etter than compared with N6B1.

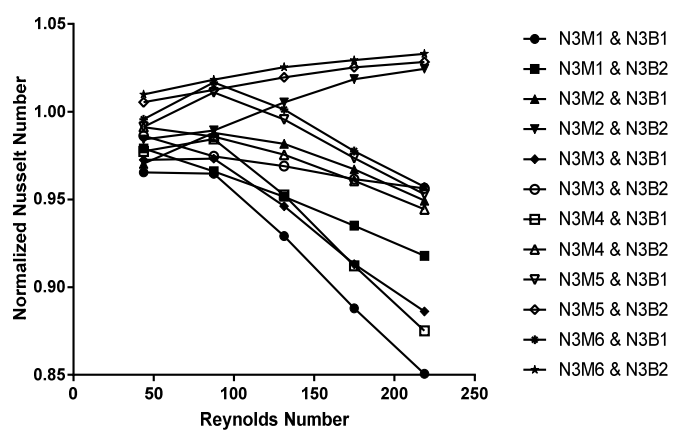

Fig 7: Nusselt Number of water-vapor of Modified Heat Exchanger (HX) with corresponding Reynolds Number of Conventional HX for $\mathrm{N}=3$

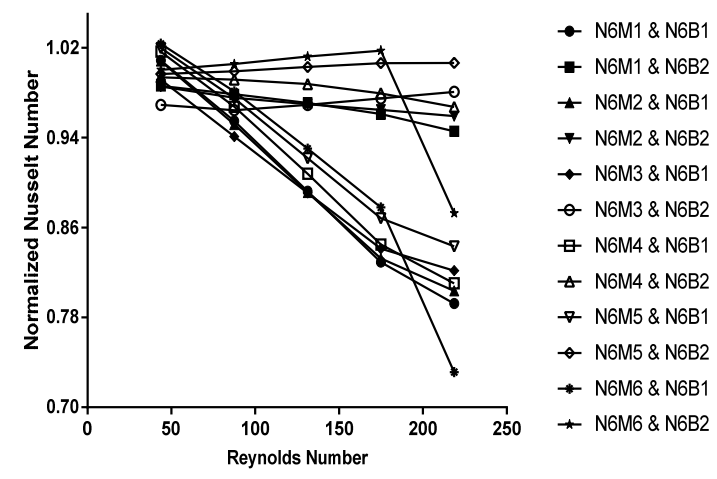

Fig 8: Nusselt Number of water-vapor of Modified Heat Exchanger (HX) with corresponding Reynolds Number of Conventional $\mathrm{HX}$ for $\mathrm{N}=\mathbf{6}$ 


\subsection{Friction factor for air}

Pressure drops are represented in terms of friction factor and inlet air velocity is taken as in terms of Re number.

From Fig 9, it is shown that friction for N3M2 increases having high fric tion factor. In contrast friction factor for N3M1 decreas es when compared with N3B2. N3M1 combination is better when compared with N3B1. N3M1 is better when compared with N3 B2 because it has lower friction co-efficient. It is shown that N3M3 is the best combination because it has a much lower friction factor. But for the N3M3 pressure drop is higher when com pared with N3B2. N3M4 also have lower pressure drop when co mpared with N3B1 than N3B2. Press ure drop de creases as velocity increases. It is shown that for N3M5 and N3M6 pressure drop reduces as velocity increases when compared with N3B1. Reverse action occurs when compared with N3B2.

From Fig 10, it is shown that N6M1 is the best combination because it has a much lower friction factor. But for the N6M1 pressure drop is higher when compared with N6 B2. N6M2 also have lower pressure drop whe n compared with N6B1 than N6B2. N6M3 and N6M4 for both cases pressure drop reduces as velocity increases when compared with N6B1. But slight in pressure drop for N6M3 when velocity crosses $2.0 \mathrm{~m} / \mathrm{s}$. For N6M5 pressure drop is higher when compared with N6B2. N6M6 also have lower pressure drop when compared with N6B1 than N6B2. Pressure drop decreases as veloci ty increases. Reverse when N6M5 and N6M6 are compared with N6B2.

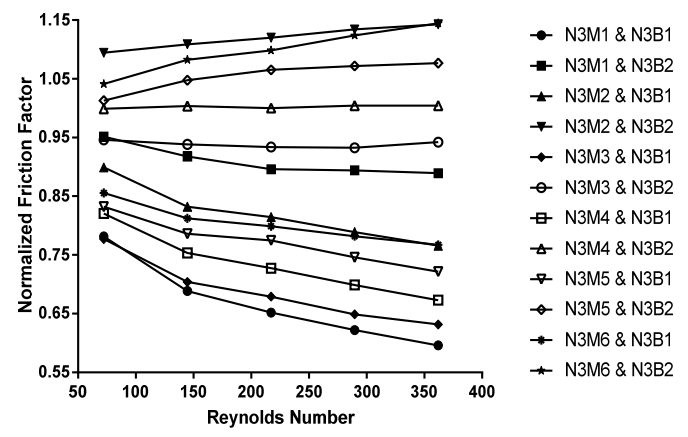

Fig 9: Normalized Friction Factor of air of Modified Heat Exchanger (HX) with corresponding Reynolds Number of Conventional $\mathrm{HX}$ for $\mathbf{N}=\mathbf{3}$

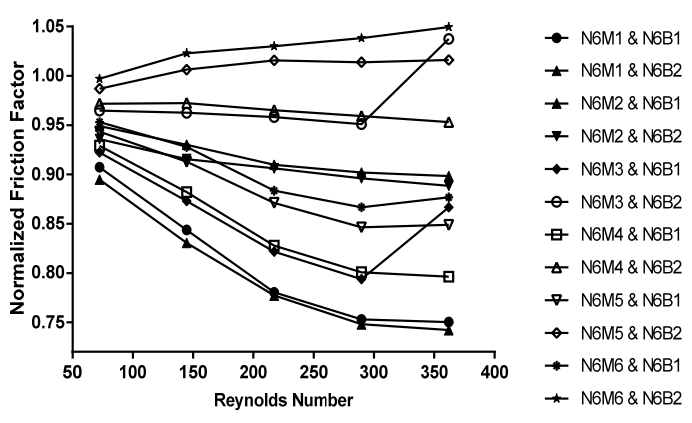

Fig 10: Normalized Friction Factor of air of Modified Heat Exchanger (HX) with corresponding Reynolds Number of Conventional $\mathrm{HX}$ for $\mathrm{N}=6$

\subsection{Friction factor for water-vapor}

Friction factor for water-vapor is estimated and plotted in terms of friction factor and Re number for number of tubes 3 and $6 \mathrm{f}$ or various combinations. From Fig 11, it is al most similar to air. N3M1 is better when com pared with N3B2 because it has lower friction co-efficient. In contrast friction factor for N3M1 decreases when compared with N3B2. N3M1 combination is better when compared with N3B1. It is observed that at low i nlet velocity for N3M3 and N3M4 value is respectively 0.85 and 0.83 but when velocity increases pressure drop reduces and respectively the values are 0.73 and 0.68 when both are compared with N3B1. N3M3 and N3M4 are compared with $\mathrm{N} 3 \mathrm{~B} 2$ friction factor values are 0.95 and 0.98 . For N3M3 pressure drops values almost equal to N3B2. N3M5 and N3M6 pressure drop reduces as velocity increas es when compared with N3B1. Friction factor for N3M5 and N3M6 at lower velocity, friction factor velocity are respectively 0.86 and 0.87 . But for higher velocity those values decrease to 0.80 and 0.78 . Reverse action occurs when compared with N3B2.

From Fig 12, it is sho wn that for N3M5 and N3M6 pressure drop reduces as velocity increases when compared with N3B1. N6M2 is one of the best combination because it has much lower friction factor having fric tion factor 0.925 . For N6M2 and N6B2 values reduce to 0.92 while it started from 0.96, N6M1 also have lower pressure drop when compared with N6B1 than N6B2.

Friction factor for N6M3 \& N6B1 red uces while friction factor for N6M3 \& N6B2 increases. It is also monitored that N6M3 \& N6B2 and N6M4 \& N6B2 pressure drop increase in a similar fashion. 


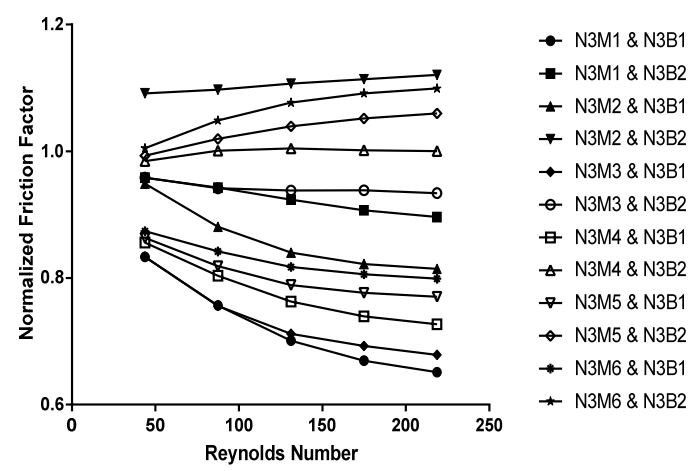

Fig 11: Normalized Friction Factor of water-vapor of Modified Heat Exchanger (HX) with corresponding Reynolds Number of Conventional $\mathrm{HX}$ for $\mathrm{N}=3$

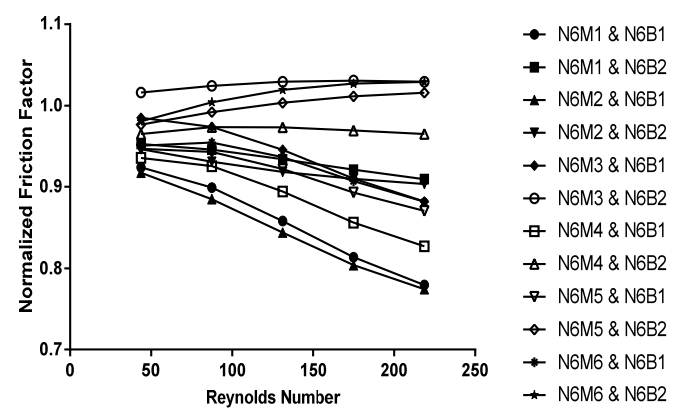

Fig 12: Normalized Friction Factor of water-vapor of Modified Heat Exchanger (HX) with corresponding Reynolds Number of Conventional $\mathrm{HX}$ for $\mathrm{N}=6$

\subsection{Temperature contour of air for various combination}

Temperature contour means the distr ibution of temperature for air. In the front portion of fin, heat transfer is large because high temp difference but at rear portion heat transfer reduce $d$ because difference of tube surface te $\mathrm{mp}$ and air is lower. Only one conventional and modified temp contour is shown here [18].

a) Geometry N3B1 $0.5 \mathrm{~m} / \mathrm{s}$

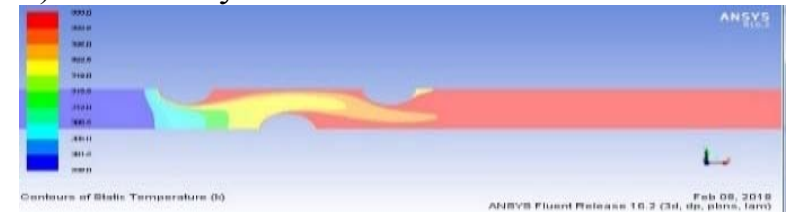

b) Geometry N3B1 $2.5 \mathrm{~m} / \mathrm{s}$

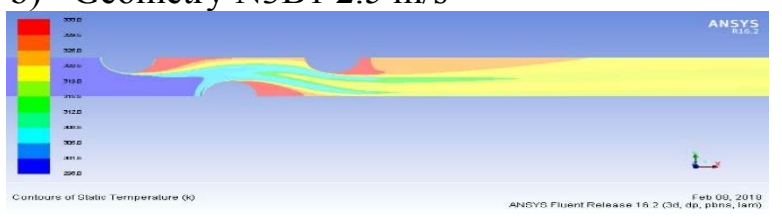

c) Geometry N3M1 $0.5 \mathrm{~m} / \mathrm{s}$

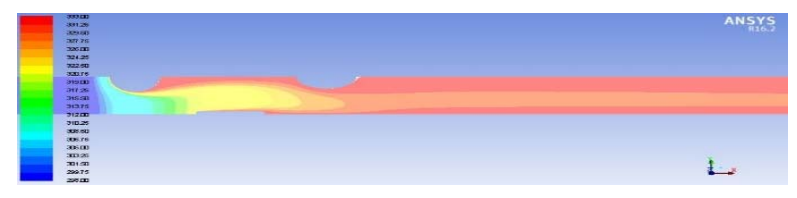

d) Geometry N3M1 $2.5 \mathrm{~m} / \mathrm{s}$

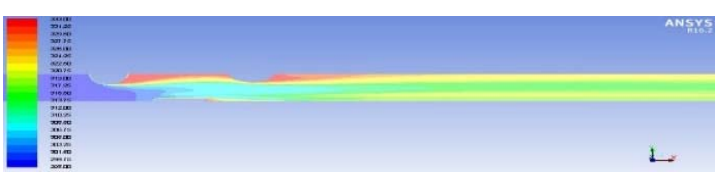

Fig 13: Temperature contour of air a) N3B1 at $0.5 \mathrm{~m} / \mathrm{s} \mathrm{b}$ ) $\mathrm{N} 3 \mathrm{~B} 1$ at $2.5 \mathrm{~m} / \mathrm{s} \mathrm{c}$ ) $\mathrm{N} 3 \mathrm{M} 1$ at $0.5 \mathrm{~m} / \mathrm{s} \mathrm{d}$ ) $\mathrm{N} 3 \mathrm{M} 1$ at $2.5 \mathrm{~m} / \mathrm{s}$

\subsection{Pressure Contour for Air}

Pressure contour of air three rows of tube as static pressure distribution. As the velocity increases pressure drop increase s. Pressure contour shown for three ro ws of tube one con ventional and another for modified heat exchanger [18].

a) Geometry N3B1 for $0.5 \mathrm{~m} / \mathrm{s}$

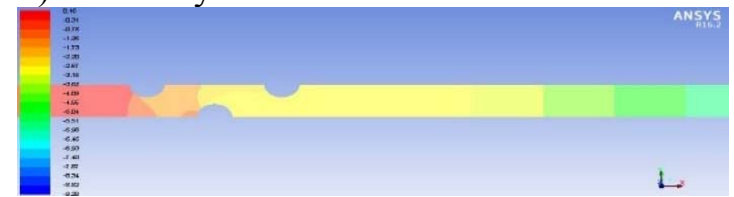

b) Geometry N3B1 for 2.5

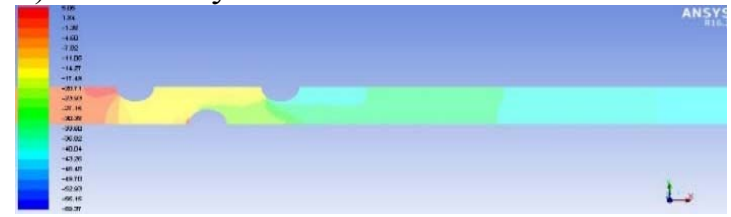

c) Geometry of N3M3 at $0.5 \mathrm{~m} / \mathrm{s}$

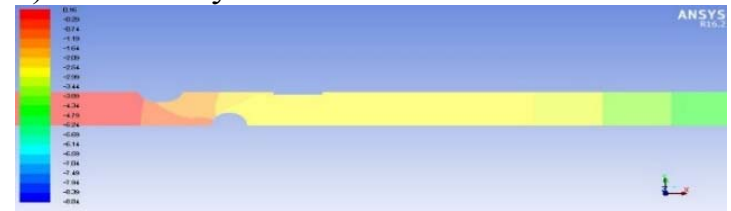

d) Geometry of N3M3 at $2.5 \mathrm{~m} / \mathrm{s}$

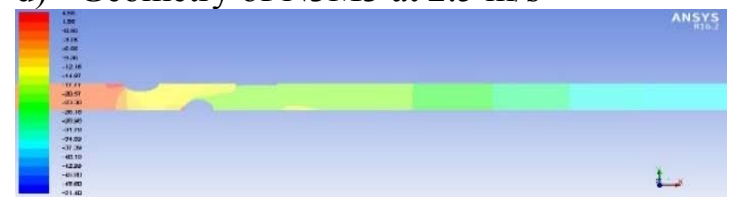

Fig 14: Pressure contour for air a) N3B1 at $0.5 \mathrm{~m} / \mathrm{s}$ b) N3B1 at $2.5 \mathrm{~m} / \mathrm{s} \mathrm{c}$ ) N3M3 at $0.5 \mathrm{~m} / \mathrm{s} \mathrm{d}$ ) N3M3 at $2.5 \mathrm{~m} /$

\subsection{Temperature contour of water-vapor}

Temperature distribution is shown for water-vapor for three rows of tube. There is little di fference in outlet temp of water-vapor than air [18]. 
a) Geometry N3B1 $0.5 \mathrm{~m} / \mathrm{s}$

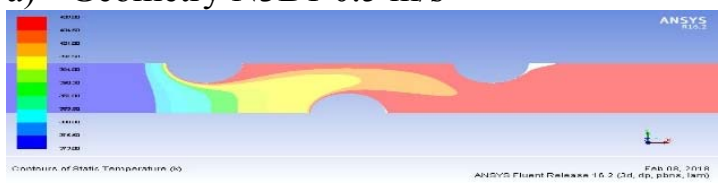

b) Geometry N3B1 $2.5 \mathrm{~m} / \mathrm{s}$

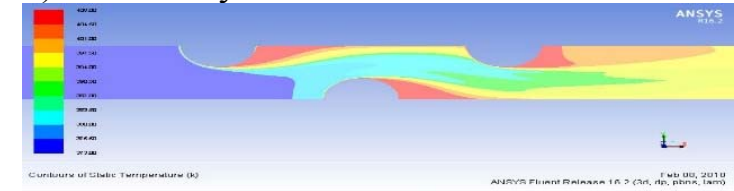

c) Geometry of N3M3 at $0.5 \mathrm{~m} / \mathrm{s}$

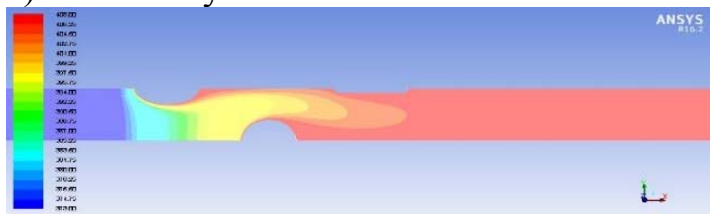

d) Geometry of N3M3 at $2.5 \mathrm{~m} / \mathrm{s}$

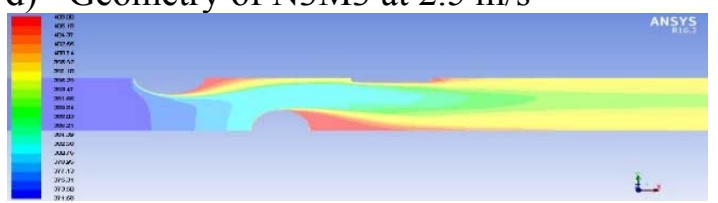

Fig 15: Temperature contour of water-vapor a) $\mathrm{N} 3 \mathrm{~B} 1$ at $0.5 \mathrm{~m} / \mathrm{s}$ b) $\mathrm{N} 3 \mathrm{~B} 1$ at $2.5 \mathrm{~m} / \mathrm{s} \mathrm{c}$ ) $\mathrm{N} 3 \mathrm{M} 3$ at 0.5 $\mathrm{m} / \mathrm{s} \mathrm{d}) \mathrm{N} 3 \mathrm{M} 3$ at $2.5 \mathrm{~m} / \mathrm{s}$

\subsection{Pressure contour of water-vapor}

Static pressure distribution are shown for watervapor are presented for one baseline ca se another for modified case [18].

a) Geometry N3B1 for $0.5 \mathrm{~m} / \mathrm{s}$

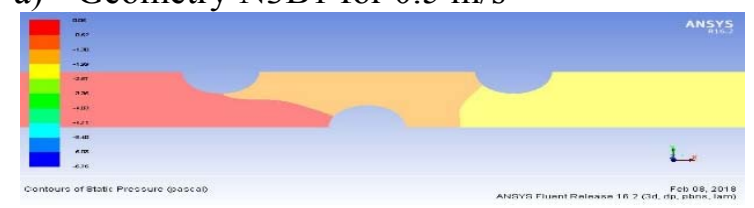

b) Geometry N3B1 for $2.5 \mathrm{~m} / \mathrm{s}$

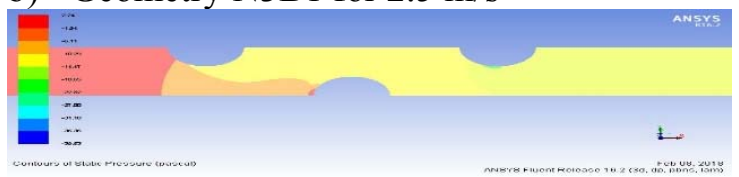

c) Geometry of N3M3 at $0.5 \mathrm{~m} / \mathrm{s}$

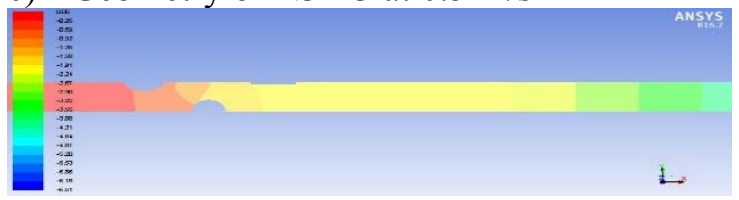

d) Geometry of N3M3 at $2.5 \mathrm{~m} / \mathrm{s}$

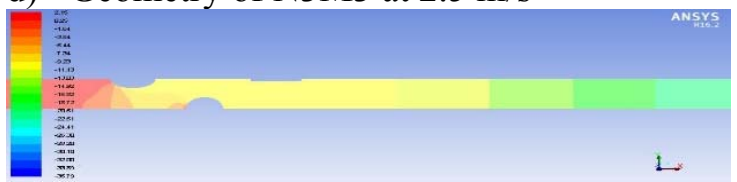

Fig 16: Pressure contour for water-vapor a) N6B1 at $0.5 \mathrm{~m} / \mathrm{s} \mathrm{b}$ ) N6B1 at $2.5 \mathrm{~m} / \mathrm{s} \mathrm{c)} \mathrm{N3M3} \mathrm{at} 0.5 \mathrm{~m} / \mathrm{s} \mathrm{d}$ ) $\mathrm{N} 3 \mathrm{M} 3$ at $2.5 \mathrm{~m} / \mathrm{s}$

\subsection{Discussion}

In this resea rch work, several co mbinations of three rows of tube with circular, elliptical and rectangular tubes have $b$ een investigated. These tubes have also been used for six rows of tube. First of all, fluid is taken at the air. Then air is replaced with water-vapor. Present si mulation has investigated with an established paper and compared with that paper in ter $\mathrm{ms}$ of pressure drop vs. velocity. It has been inspected from the graph of nor malized $\mathrm{Nu}$ num ber and Re number that heat transfer for several geo metries gives a high heat transfer rate than convention al grouped circular and elliptical tubes. For example for air geometry N3M6 performs better than convention grouped elliptical tubes. It has been ob served that as velocity increases pressure drop also increase s. In contrary, friction factor decre ases as velocity has increased being friction factor a nd velocity anti-proportional. For example, friction factor for geometry N3B1 at velocity $0.5 \mathrm{~m} / \mathrm{s}$ was 0.95 and for velocity $2.5 \mathrm{~m} / \mathrm{s}$ it was 0.37 . F or verification, the result has been compared with the heat transfer correlation for tube ban ks. $1.41 \%$ of error occurred with the actual co-relation result. The results show that, at low inlet velocity for air, modified heat exchanger 4 and 6 have $7.54 \%$ and $4.59 \%$ increase in heat tra nsfer co-efficient from the conventional circular tube heat exchanger when $\mathrm{N}=3$. But heat transfer co-efficient is decreases $2.42 \%$ for $\mathrm{m}$ odified heat exchanger 3 from grouped elliptical tube when $\mathrm{N}=3$. When the number of tubes are six, for all the modified heat exchangers heat transfer co-efficient decreas e almost 2-3\% from grouped circular and elliptical tube heat exchanger. In case of $w$ ater-vapor, modified heat exchanger 1 and 2 have $3.48 \%$ decrease in heat transfer from conventional circular tube heat exchanger. Also modified heat exchanger 1 and 2 have $2.11 \%$ decrease in heat transfer from conventional elliptical tube heat exchanger. On the contrary, at high i nlet velocity, modified heat exchanger 2 have $10.45 \%$ higher from grouped elliptical $t$ ube heat exchanger, for modified heat exchanger 6 gives $5 . \quad 80 \%$ higher heat transfer fro $\mathrm{m}$ grouped elliptical tube heat exchanger when $\mathrm{N}=3$. This has been due to flow separation point of the fluid over the tube surface. As long as flow adhered to the tube surface, fluid was heated up. Vortex ge nerator could have been used to delay flow separation. 


\section{Conclusion}

Air side and water-vapor heat transfer characteristics for diffe rent arrangements of circular, elliptical and rectangular tubes, has been numerically investigated in the laminar flow region for multiple rows. The result is presented as follows: The heat transfer enhancement is being quite good for air, the geometry N3M2 and N3M5 than the grouped elliptical tube heat exchanger in case of three rows of tube. On the other hand, for six rows tube N6M3 and N6M6 has performed better than the grouped $c$ ircular tube. In case of water-vapor, the heat exchanger with geom etry N3M1 and N3M2 has been performed quite better than both ci rcular and elliptical tubes. $\mathrm{Si}$ milar performance has been observed for geometry N6M5 and N6M6 when compared with conventional heat exchanger. The frictional resistance of air for geometry N3M1 and N3M3 when compared with grouped circular tube. For six rows of tube, N6M5 a cts better than circular tube and N6M6 also performs better than elliptical tubes alone. The frictional resist ance for watervapor N3M1 N3M3 and N3M4 all has performed better than grouped circul ar and ellipti cal tubes. N6M1 and N6M2 has acted better than grouped circular tube heat exchanger.

\section{References}

[1] M. N. Ozi sik, 1985. “ Heat Transfer a Basic Approach", International Edition, 1985, M cGrawHill Book Company. ISBN 0- 07-066460-9.

[2] T. Kuppan, "Design of a heat exchanger handbook", Third Edition, Marcel Dekk er Inc. 2010.

[3] F.P. Incropera, D.P Dewitt, "Fundamentals of Heat \& Mass Transfer" Seventh Edition, 2013, ISBN 13 978-0470-50197-9

[4] Xie G, Wang Q, Sunden B, "Parametric study and multiple correlations on air-side heat transfer and friction characteristics of fin-and-tube heat exchangers with a large number of large-diameter tube rows", Applied Thermal Engineering 2009; 29:1-16

[5] Guo ZY, Li DY, Wang BX, "A novel concept for convective heat transfer enhancement", International Journal Heat Mass Tran sfer1998; 41:2221-25.

[6] Li J, Wang S, Chen J, Lei YG, "Numerical study on a slit fin-and-tube heat exchanger with longitudinal vortex generators", International Journal Heat Mass Transfer 2011; 54:1743-51

[7] Jayavel S, Sh aligram Tiwari, " Effect of tube spacing on heat transfer performance of staggered tube bundles in presence of vortex generators", Journal Enhance Heat Transfer 2010; 17:271-91.

[8] Jayavel S, Shalig ram Tiwari, "Effect of vortex generators and integral splitter plate on heat transfer and pressure drop for laminar flow past channel-confined tube banks", Heat T ransfer Engineering 2010; 31:383-94.

[9] Jayavel S, Sh aligram Tiwari, “ Finite volume algorithm to study the effect of tube separation in flow past channel confined tube banks", Engineering Applied Fluid Mechanics 2010; 4:3957.

[10] Wu JM, Tao WQ, "Impact of delta winglet vortex generators on the performance of a novel fin-tube surfaces with two rows of tubes in different diameters", Energy Conversion Manage 2011; 52:2895-901.

[11] Xie G, Wang Q, Sunden B, "Parametric study and multiple correlations on air-side heat transfer and friction characteristics of fin-and-tube heat exchangers with large number of large-diameter tube rows", Applied Thermal Engineering 2009; 29:1-16.

[12] Lu CW, Huang JM, Nien W C, Wang CC, “A numerical investigation of the geometric effects on the performance of plate finned-tube heat exchanger", Energy Conversion Manage 2011; 52:1638-43.

[13] Lotfi B, Sunden B, Wang QW, “An investigation of the thermo-hydraulic performance of the smooth wavy fin-and-elliptical tube heat exchangers utilizing new type vortex generators". Applied Energy. 2016; 162: 1282-1302.

[14]Zukauskas, A. "Heat transfer from tubes in cross flow, in Handbook of single phase convective heat transfer", Wiley International science, 1987.

[15] Rocha LAO, Saboya FE M, Vargas J VC, “A comparative study of elliptical and circular sections in one- and two-row tubes and plate fin heat exchangers", International Journal Heat Fluid Flow 1997; 18:247-252.

[16]R. Deepakkumar, S. Jayav el, "Air side performance of finned-tube heat exchanger with combination of circular and elliptical tubes" Applied Thermal Engineering, 2017, S13594311(16)31949-4

[17] Wang CC, Chi KY, Chang CJ, "Heat transfer and friction characteristics of plain finned- tube heat exchangers, part II: Correlation", International Journal Heat Mass Transfer 2000; 43:2693-7

[18] H. Hasan, "Numerical Investigation of Finned-tube Heat Exchanger with Circular, Elliptical \& Rectangular Tubes", A Project of B.Sc. Engg. (ME) degree, Department of Mechanical Engineering, Khulna University of Engineering \& Technology, Khulna-9203, Bangladesh, February 2018. 